\title{
Gastric volvulus: a rare cause of gastric outlet obstruction in pediatric age
}

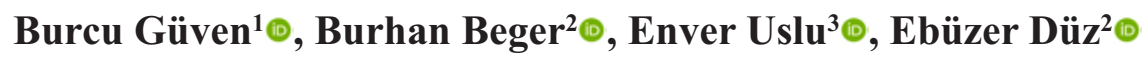 \\ ${ }^{1}$ Department of Pediatric Gastroenterology, Yüzüncü Yll University School of Medicine, Van, Turkey \\ ${ }^{2}$ Department of Pediatric Surgery, Yüzüncü Yıl University School of Medicine, Van, Turkey \\ ${ }^{3}$ Department of Pediatrics, Yüzüncü Yıl University School of Medicine, Van, Turkey
}

DOI: $10.18621 /$ eurj.407805

\begin{abstract}
Gastric volvulus is a rare disease in the pediatric population. Symptoms may change depending on the type of the disease, rotation degree, and the etiology. Early diagnosis and treatment are important especially in the acute type of the gastric volvulus. Here, it was presented a 10-month-old girl with non-bilious vomiting and abdominal distention in last 2 days, diagnosed gastric volvulus.
\end{abstract}

Keywords: gastric volvulus, pediatric

Received: March 19, 2018; Accepted: July 25, 2018; Published Online: July 26, 2018

G astric outlet obstruction is a rare condition encountered in the pediatric age group of 2-5 years and its incidence is $1: 1000$ [1]. Due to the obstruction of the passage of the gastric content to the duodenum, persistent non-bilious vomiting followed by dehydration and electrolyte imbalance develops. The most common cause is idiopathic hypertrophic pyloric stenosis. Other rare causes are distal gastric antral webs, gastric duplication cysts, gastric polyps, neoplasms, Crohn's disease, bezoar, complications of the gastrostomy tube, malrotations and volvulus [1].

Gastric volvulus is a rare disease, especially in children. It was first defined by Berti in 1866 [2]. In a review published in 1980, 51 patients were described and $52 \%$ of these patients were under one year of age and $26 \%$ were less than 1 month old [2]. Less than 600 pediatric patients with gastric volvulus were reported until 2008 [3]. Although its incidence is unknown, it is evenly distributed regarding the gender and race [4].

\section{CASE PRESENTATION}

A 10-month-old girl was admitted to the clinic with the complaints of non- bilious vomiting and abdominal distention in last 2 days. She had vomited everything she had eaten. There was no special finding in her medical and family history and distension was observed in the left upper abdominal quadrant during the physical examination. Abdominal plain radiography revealed that left hemidiaphragm was elevated, the stomach was dilated and air passage to distal was obstructed (Figure 1). Abdominal ultrasonography showed a thick fluid in the gastric lumen and intense echogenic appearance partly with air-fluid levels. Esophagogastroduodenoscopy revealed abundant fluid in the corpus and a changed gastric configuration. Abdominal computed tomography showed that the gastroesophageal junction was dislocated and the pylorus moved to 


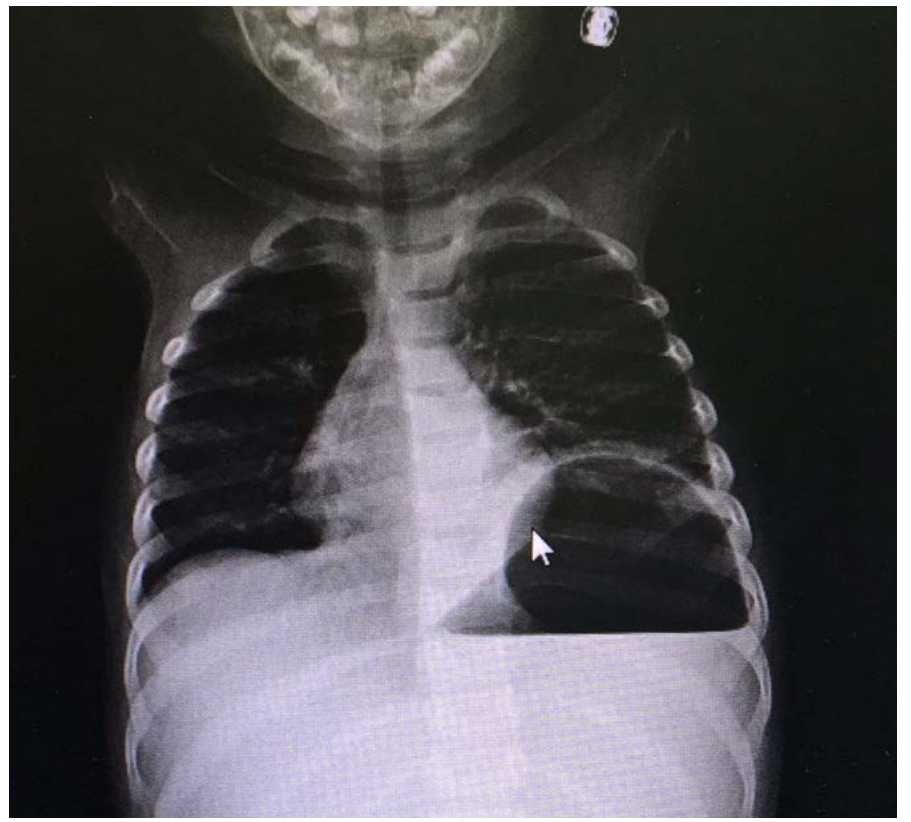

Figure 1. Abdominal plain radiography revealed that the left hemidiaphragm was elevated, the stomach was dilated and air passage to distal was obstructed.

anterior of the abdominal midline (Figure 2). In light of these findings, it was considered mesentero-axial gastric volvulus and referred to the pediatric surgery. During the operation, it was observed that the stomach had rotated clockwise about $360^{\circ}$ in the longitudinal position. Gastric volvulus reduction and anterior gastropexy were performed. There was no problem with her follow-up.

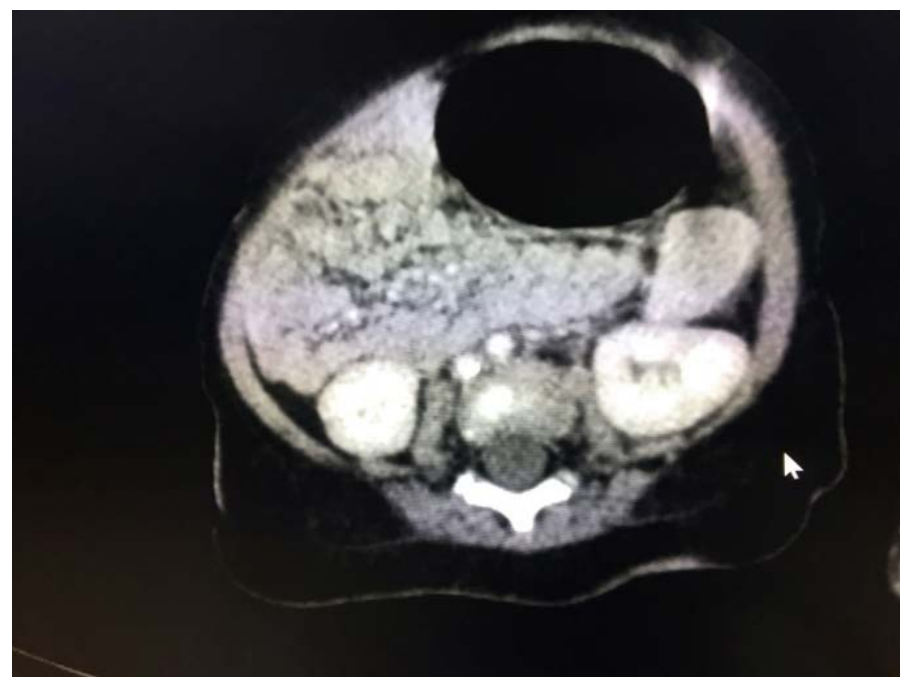

Figure 2. Abdominal computed tomography showed that the gastroesophageal junction was dislocated and the pylorus moved to anterior of the abdominal midline

\section{DISCUSSION}

Gastric volvulus is defined as a partial or complete rotation of the stomach of more than $180^{\circ}$. There are 3 types: organo-axial, mesentero-axial and combined. Organo-axial type is seen in $50-60 \%$ of the cases. In organo-axial volvulus, the stomach rotates around the longitudinal axis connecting the gastro-esophageal junction with the pylorus. The greater curvature of the stomach lies superior to the lesser curvature. In the mesentero-axial type, the stomach rotates around the transverse axis and pylorus lies superior to the gastroesophageal junction at anterior side. In the combined type, the rotation occurs in both longitudinal and transverse axes.

The stomach is normally fixed by four ligaments. These are gastrocolic, gastrohepatic, gastrophrenic and gastrosplenic ligaments. Agenesis, elongation or disruption of these ligaments leads to primary gastric volvulus [5]. The causes of the secondary gastric volvulus are a diaphragmatic hernia, asplenia, pyloric stenosis, phrenic nerve palsy or esophageal atresia operations [6].

Gastric volvulus causes gastric outlet obstruction. This obstruction may be acute, recurrent, intermittent or chronic. Acute gastric volvulus is a life-threatening condition, which should be diagnosed and treated as quickly as possible. These patients admit to the clinic with persistent, no bilious vomiting, epigastric pain, and distension. More rarely, they may present with dyspnea, cyanosis or hematemesis. Due to gastric outflow obstruction, hypochloremic hypokalemic alkalosis and dehydration can develop. As a result of the delayed diagnosis, $5-28 \%$ of the patients can present with ischemia, necrosis or perforation [7]. Gastro-intestinal bleeding and septic shock may be encountered in these patients. The mortality in gastric volvulus with necrosis and perforation increases up to $50 \%$ [8]. The symptoms are usually non-specific in chronic gastric volvulus. These patients often apply to the clinic with distention related to the eating, epigastric pain, vomiting, and weight loss [9]. Usually, anatomical defects are associated with the acute type. On the other hand, the loss of the ligament laxity is in the chronic gastric volvulus [10].

In chronic gastric volvulus patients with nonspecific symptoms, the diagnosis may be delayed. An incidental diagnosis is common on an upper 
gastrointestinal contrast study performed with reflux symptoms. In contrast study, it is usually detected a distended stomach lying in a horizontal and upright position. Some clues in the plain radiographs indicating gastric volvulus include air-fluid level in the epigastrium, abnormal gastric double-bubble sign and epigastric distention despite the presence of nasogastric tube [9]. Abdominal ultrasonography and upper gastrointestinal endoscopy may be not useful regarding the diagnosis [10]. Computed tomography is helpful in confirming the diagnosis of gastric volvulus. Although ionizing radiation has disadvantages, it is valuable in showing the anatomy [7].

The treatment depends on the type of the gastric volvulus. In acute gastric volvulus, following the decompression with a nasogastric tube, the patient must be referred to the acute surgery. In order to assure the normal gastric configuration, gastropexy is performed. Anatomical problems like a hiatal hernia may be corrected simultaneously during the operation. Untreated acute gastric volvulus may lead to strangulation, necrosis, and perforation [11]. The mortality rate is twice high in acute gastric volvulus compared to the chronic volvulus [5]. Secondary gastric volvulus may recover without gastropexy if the predisposing factors are eliminated [6]. In patients with chronic volvulus, the conservative treatments such as prone position (with head slightly up), prokinetics, antisecretory drugs and diet modification may be tried [10]. Al-Salem [12] reported that the conservative treatment was beneficial in 11 chronic volvulus patients with mild-moderate symptoms.

\section{CONCLUSION}

In conclusion, gastric volvulus is a rare disease in the pediatric population. Symptoms may change depending on the type of the disease, rotation degree, and the etiology. Early diagnosis and treatment are important especially in the acute type of the gastric volvulus.

\section{Informed consent}

Written informed consent was obtained from the patient's family for publication of this case report and any accompanying images.

\section{Conflict of interest}

The authors declared that there are no potential conflicts of interest with respect to the research, authorship, and/or publication of this article.

\section{REFERENCES}

[1] Otjen JP, Iyer RS, Phillips GS, Parisi MT. Usual and unusual causes of pediatric gastric outlet obstruction. Pediatr Radiol 2012;42:728-37.

[2] Cameron AEP, Howard ER. Gastric volvulus in childhood. J Pediatr Surg 1987;22:944-7.

[3] Joshi M, Parelkar S, Sanghvi B, Agrawal A, Mishra P, Pradeep SH. Gastric volvulus in children: experience of 6 years at a tertiary care centre. Afr J Paediatr Surg 2010;7:2-4.

[4] Rashid F, Thangarajah T, Mulvey D, Larvin M, Iftikhar SY. A review article on gastric volvulus: a challenge to diagnosis and management. Int J Surg 2010;8:18-24.

[5] Cribbs RK, Gow KW, Wulkan ML. Gastric volvulus in infants and children. Pediatrics 2008;122:752-62.

[6] Mirza B, Ijaz L, Sheikh A. Gastric volvulus in children: our experience. Indian J Gastroenterol 2012;31:258-62.

[7] Trecroci I, Morabito G, Romano C, Salamone I. Gastric volvulus in children--a diagnostic problem: two case reports. J Med Case Rep 2016;10:138.

[8] Farag S, Fiallo V, Nash S, Navab F. Gastric perforation in a case of gastric volvulus. Am J Gastroenterol 1996;91:1863-4.

[9] Duman L, Savas MC, Büyükyavuz BI, Akcam M, Sandal G, Aktas AR. Early diagnostic clues in neonatal chronic gastric volvulus. Jpn J Radiol 2013;31:401-4.

[10] Porcaro F, Mattioli G, Romano C. Pediatric gastric volvulus: diagnostic and clinical approach. Case Rep Gastroenterol 2013;7:63-8.

[11] Carter R, Brewer LA, Hinshaw DB. Acute gastric volvulus. A study of 25 cases. Am J Surg 1980;140:99-106.

[12] Al-Salem AH. Acute and chronic gastric volvulus in infants and children: who should be treated surgically? Pediatr Surg Int 2007;23:1095-9. 